Pacific Journal of Mathematics

LOCALLY COMPACT GROUPS ACTING ON TREES 


\section{LOCALLY COMPACT GROUPS ACTING ON TREES}

\section{ROGER ALPERIN}

Following Serre's original description of groups having the fixed point property for actions on trees, Bass has introduced the notion of a group of type $\mathrm{FA}^{\prime}$. Groups of type $\mathrm{FA}^{\prime}$ can not be nontrivial free products with amalgamation. We show that a locally compact (hausdorfi) topological group with a compact set of connected components is of type $\mathrm{FA}^{\prime}$. Furthermore, any locally compact group which is a nontrivial free product with amalgamation has an open amalgamated subgroup.

1. A group $G$ is called an amalgam if it is a free product with amalgamation of subgroups $A$ and $B$ along $C$, i.e., $G=A_{C}^{*} B$, so that $C \neq A, C \neq B$.

If a group $G$ acts without inversions on a tree so that it has a fixed vertex we say $G$ has property FA on $X$. Serre has introduced the notion of a group of type FA. We say that $G$ is of type FA if $G$ has property FA whenever it acts on a tree. The following theorem characterizes $G$ group theoretically.

THEOREM 1 (Serre). A group $G$ is of type FA if and only if it satisfies the following conditions:

(1) $G$ has no infinite cyclic quotient.

(2) $G$ is not an amalgam.

(3) $G$ is not the union of any sequence

$$
G_{0} \varsubsetneqq G_{1} \varsubsetneqq G_{2} \cdots \varsubsetneqq G_{n} \varsubsetneqq \cdots
$$

of its proper subgroups.

This theorem was originally formulated by Serre for countable groups [6, Theorem 15;2, Theorem 3.2]. Bass has introduced the notion of a group of type $\mathrm{FA}^{\prime}$. In order to formulate this we introduce the ends of a tree $X$. Consider the collection $\mathscr{L}$ of halflines of $X: L \in \mathscr{L}$ is isometric to the standard half-line

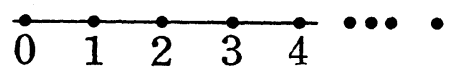

The ends of $X$ is the set of equivalence classes $\mathscr{E}$ of $\mathscr{L}$ under the equivalence relation $\sim$ :

$$
L \sim M \text { iff } L \cap M \text { is a half-line . }
$$


Notice that if $e, f \in \mathscr{E}, e \neq f$, we can choose representatives $L \in e$, $M \in f$ so that $L \cup M$ is a doubly infinite line of $X$ denoted $(e, f)$. If $G$ acts as a group of isometries on $X$ then it also acts on the set $\mathscr{L}$ of half-lines of $X$ and the set $\mathscr{E}$ of ends of $X$. If $L \in \mathscr{L}, g \in G$ we say $L$ is neutral, repulsing or attracting for $g$ if $g L=L$ (i.e., pointwise fixed), $g L \supsetneq L$, or $g L \varsubsetneqq L$ respectively. If $L$ contains a half-line $L^{\prime}\left(L-L^{\prime}\right.$ is finite) which is neutral, repulsing or attracting for $g$ then we say $L$ is almost neutral, repulsing or attracting for g. An end $e \in \mathscr{E}$ is neutral, repulsing or attracting for $g \in G$ if it possesses a representative half-line which is so for $g$. Denote the ends which are fixed by $G(g e=e, \forall g \in G)$ by $\mathscr{f}^{G}$.

We can now formulate the property $\mathrm{FA}^{\prime}$.

THEOREM 2. Suppose $G$ acts without inversion on the tree $X$. The following conditions are equivalent.

(i) Each element of $G$ has a fixed vertex.

(ii) Each finitely generated subgroup of $G$ has a fixed vertex.

(iii) There is either a fixed vertex for $G$ or a neutral fixed end.

Proof. The implication (i) $\Rightarrow$ (ii) is proved by Serre [6, Corollary 3 to Proposition 26]. The implication (i) $\Rightarrow$ (iii) is proved by Tits [8, Corollary 3.4]. The implications (ii) $\Rightarrow$ (i) and (iii) $\Rightarrow$ (i) are obvious.

If $G$ satisfies the equivalent conditions of Theorem 2 for a given action without inversions on a tree $X$ then we shall say $G$ has property $\mathrm{FA}^{\prime}$ on $X$. This property has been further analyzed by Bass [2, Propositions 1.6, 3.7]. In case $G$ has property $\mathrm{FA}^{\prime}$ on $X$ and has no fixed vertex then there is a half-line $L$ with vertices $\left(v_{n}\right), n \geqq 0$, so that $G_{v_{n}} \subset G_{v_{n+1}}, n \geqq 0$, and

$$
G=\bigcup_{n \geq 0} G_{v_{n}} \text {. }
$$

We say that $G$ is of type $\mathrm{FA}^{\prime}$ if $G$ has property $\mathrm{FA}^{\prime}$ whenever it acts on a tree.

THEOREM 3 (Bass). A group $G$ is of type $\mathrm{FA}^{\prime}$ if and only if it satisfies the following conditions:

(1) $G$ has no infinite cyclic quotient.

(2) $G$ is not an amalgam.

One obtains information about homomorphisms from a group $G$ of type $\mathrm{FA}$ or $\mathrm{FA}^{\prime}$ to amalgams using the next propositions.

Proposition 1 (Serre [6, Proposition 21]). If $G$ is a group of 
type FA and $\varphi: G \rightarrow A_{C}^{*} B$ is a homomorphism to an amalgam then $\varphi(G)$ is contained in a conjugate of $A$ or $B$.

Proposition 2. If $G$ is a group of type $\mathrm{FA}^{\prime}$ and $\varphi: G \rightarrow A^{*} B$ is a homomorphism then $\varphi(G)$ is contained in a conjugate of $A$ or $B$.

Proof. First notice that a homomorphic image of type $\mathrm{FA}^{\prime}$ is also of type $\mathrm{FA}^{\prime}$. Thus $\varphi(G)$ acts without inversions on the tree $X$ for $A^{*} B$. Using condition iii) of Theorem $2, \varphi(G)$ has a fixed point and consequently $\varphi(G)$ is contained in a conjugate of $A$ or $B$, or there is a neutral fixed end for $\varphi(G)$. However, the edge stabilizers for this fixed end are trivial since $A^{*} B$ has no amalgamation; this is impossible and consequently $\varphi(G)$ has a fixed point.

2. If $H$ is a normal subgroup of type FA of a group $G$ and $G / H$ is of type $\mathrm{FA}^{\prime}(\mathrm{FA})$ then $G$ is of type $\mathrm{FA}^{\prime}(\mathrm{FA})$. To see this notice that if $G$ acts on a tree $X$ and $K$ is a finitely generated subgroup of $G$ then $L=K \cap H$ has a fixed tree $X^{L}$ and thus the finitely generated subgroup $K H / H \cong K / L$ of $G / H$ acts on $X^{L}$ with a fixed vertex which is then fixed by $K$. Also, if $G$ contains a subgroup of finite index $H$ of type $\mathrm{FA}^{\prime}$ then $G$ is also of type $\mathrm{FA}^{\prime}$. Indeed, if $K$ is a finitely generated subgroup of $G$ then $L=K \cap H$ is a finitely generated subgroup of $H$; without loss of generality we may assume $H$ is normal and thus the finite group $K / L$ has a fixed point for its action on $X^{L}$.

Based on some remarks of Tits $[7 ; \S 2.3]$ we shall show that every extension of groups of type $\mathrm{FA}^{\prime}$ is again of type $\mathrm{FA}^{\prime}$. For this we shall need some further comments on ends. We suppose that a group $G$ is acting without inversions on a tree $X$.

Proposition 3. Let $e \in \mathscr{E}^{a}$. Any half-line $L \in e$ is almost neutral, repulsing or attracting for $g \in G$.

Proof. Given $g \in G$ and $L \in e$. Let $P$ be the initial vertex of $L$. If $g P \in L$ then $L$ is neutral or attracting for $g$. If $g P \notin L$ there are two possibilities: (1) The geodesic from $P$ to $g P$ meets $L$ only at $P$ or (2) The geodesic from $P$ to $g P$ meets $L$ at a vertex $Q \neq P$. In the first case $L$ is repulsing for $g$. In the second case let $L^{\prime}$ be the half-line contained in $L$ starting at $Q$.

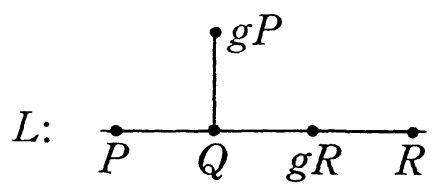


If $R \in L^{\prime}$ then $g R$ belongs to the geodesic from $g P$ to $Q$ for only finitely many $R$. Thus there is a half-line $L^{\prime \prime} \subset L^{\prime}$ so that $g L^{\prime \prime} \subset L^{\prime}$. Choose $R \in L^{\prime \prime}$ so that $Q$ belongs to the geodesic from $P$ to $R$ and $g Q$ belongs to the geodesic from $g P$ to $g R$. If $g Q$ belongs to the geodesic from $g P$ to $Q$ then $L^{\prime}$ is repulsing or neutral for $g$; otherwise, $L^{\prime}$ is attracting for $g$. It is now easy to see that if one half-line $L \in e$ is almost neutral, almost repulsing or almost attracting for $g$ then so is every half-line in $e$; viz. if $g$ has a fixed point on $L$ in $e$ then it must be almost neutral for $g$.

Proposition 4. If $G$ has a neutral fixed end e then either $\mathscr{C}^{G}=$ $\{e\}$ or there is a doubly infinite line of fixed points for $G$.

Proof. Suppose $f$ is a repulsing or attracting end for $g \in G$. Let $P \in e$ so that $g P=P$ and choose $L \in f$ on which $g$ is repulsing or attracting starting at $Q$.

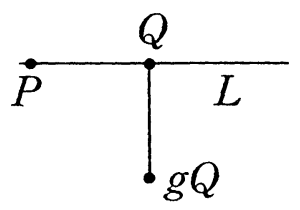

This is impossible since the length of the geodesic from $P$ to $Q$ is different from that of $g P$ to $g Q$. Thus any other fixed end $f$ for $G$ must be a neutral fixed end. Choose representative $L \in e, M \in f$ so that $L \cup M$ is a double infinite line.

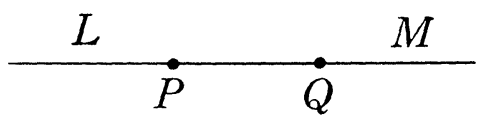

Thus for each $g \in G$ there exists $P \in L, Q \in M$ fixed by $g$; hence the doubly infinite $L \cup M$ is fixed identically for all $g \in G$.

From the above remarks we see that every half-line in $e \in \mathscr{E}^{G}$ is one of the mutually exclusive alternatives for a given $g \in G$. We can then define $v_{e}: G \rightarrow \boldsymbol{Z}$ for a fixed end $e$ as follows

$$
v_{e}(g)= \begin{cases}0 & \text { if } e \text { is neutral for } g \\ \min |L-(L \cap g L)| & \text { if } e \text { is attracting for } g \\ L \in e, L \text { attracting for } g & \\ -\min |g L-(L \cap g L)| & \text { if } e \text { is repulsing for } g .\end{cases}
$$

Theorem 4. For each fixed end $e \in \mathcal{C}^{\circ}$ there is a canonical 
homomorphism

$$
v_{e}: G \longrightarrow Z
$$

with the property that $v_{e}(g)=0$ if and only if $e$ is neutral for $g$ and $L^{g} \neq \varnothing$ for all $L \in e$.

Proof. To see that $v_{e}$ is a homomorphism let $g_{1}, g_{2} \in G$ achieve their $v_{e}$ value on $L_{1}, L_{2}$ respectively and let $g_{1} g_{2}$ achieve its $v_{e}$ value on $L$. Consider the half-line $g_{2}^{-1} L_{1} \cap L_{2} \cap L$ starting at $P$.

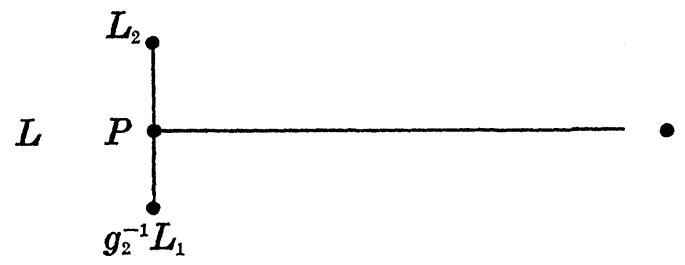

Now $P \in L_{2}$ and thus $g_{2} P \subset L_{1}$ so $P$ has moved $v_{e}\left(g_{2}\right)$ under the action of $g_{2}$. However $g_{1}\left(g_{2} P\right) \subset L_{1}$ so that $g_{2} P$ has moved $v_{e}\left(g_{1}\right)$ under the action of $g_{1}$. Thus $P$ has moved $v_{e}\left(g_{1}\right)+v_{e}\left(g_{2}\right)$ under the action of $g_{1} g_{2}$; however $P \in L$ so $P$ moves $v_{e}\left(g_{1} g_{2}\right)$ under the action of $g_{1} g_{2}$, so that

$$
v_{e}\left(g_{1} g_{2}\right)=v_{e}\left(g_{1}\right)+v_{c}\left(g_{2}\right) .
$$

If $v_{e}(g)=0$ then $e$ is neutral for $g$ and $L_{g} \neq \varnothing$ for some $L \in e$. Moreover, since $g$ fixes identically a half-line $L^{\prime} \subset L$ then it must have a fixed point on every half-line in $e$. Conversely if $g$ has a fixed point on some $L \in e$ then $L$ is almost neutral for $g$ and $e$ is neutral for $g$; thus $v_{e}(g)=0$.

CoROllary 1. If for a given action of $G$ on a tree $X$ a normal subgroup $H$ has a unique neutral fixed end $e$ then either $e$ is a neutral fixed end for $G$ or there is a nontrivial homomorphism $v: G / H \rightarrow Z$.

Proof. It is easy to see that $e$ is a fixed end for $G$; viz. suppose $g e=f \in \mathscr{E}$, then for $h \in H$

$$
e=g^{-1} h g e=g^{-1} h f .
$$

Thus $f=g e=h f$ and by uniqueness $f=e$. Thus from the theorem above $v_{e}: G \rightarrow \boldsymbol{Z}$ factors through a homomorphism $v: G / H \rightarrow Z$ since $e$ is neutral for $H$.

COROLlaRY 2. If $G$ has a normal subgroup $H$ so that $H$ and 
$G / H$ have property $\mathrm{FA}^{\prime}$ then $G$ has property $\mathrm{FA}^{\prime}$.

Proof. Let $G$ act on a tree $X$. If $H$ has a fixed point then there is an action of $G / H$ on $X^{H}$. Since $G / H$ has property $\mathrm{FA}^{\prime}$ we can find a fixed point for $g \in G$ by finding a fixed point for $g H$ on $X^{H}$. If $H$ has no fixed points on $X$ then it has a neutral fixed end and thus since $G / H$ has no homomorphism to $Z$ this neutral fixed end for $H$ is also a neutral fixed end for $G$.

COROLLARY 3. Suppose that $G$ acts without inversions on a tree $X$. If $G$ is generated by a set $S$ with $X^{s} \neq \varnothing$ for all $s \in S$ then either $G$ has no fixed end or a fixed end is neutral.

Proof. If $G$ has a fixed end $e$ then any $s \in S$ has a fixed point lying on some half-line $L \in e$; thus $v_{e}(s)=0$. It follows immediately from the theorem then that $v_{e}$ is trivial and consequently that $e$ is neutral for $G$.

A nonempty collection of subgroups $\mathscr{N}^{-}=\left\{N_{\alpha} \mid \alpha \in \mathscr{A}\right\}$ of a group $G$ is called a normal filtering family if

(1) given $\alpha, \beta \in \mathscr{A}, \exists \gamma \in \mathscr{A}$ so that $N_{\gamma} \subset N_{\alpha} \cap N_{\beta}$ and

(2) given $\alpha \in \mathscr{A}, g \in G, \exists \beta \in \mathscr{A}$ so that $N_{\beta} \subset g N_{\alpha} g^{-1}$.

(These are the conditions that guarantee $G$ is a topological group with $\mathscr{N}$ as a fundamental system of open subgroups.)

Proposition 5. Suppose that $G$ acts without inversions on a tree $X$ such that $\mathscr{E}^{G}=\varnothing$. If $\mathscr{N}$ is a normal filtering family of subgroups of $G$ having property $\mathrm{FA}^{\prime}$ on $X$ then some $N \in \mathscr{N}$ has a fixed point.

Proof. Suppose by way of contradiction that no $N \in \mathscr{N}$ has a fixed point; it follows then from the $\mathrm{FA}^{\prime}$ property that each $N_{\alpha} \in$ $\mathscr{N}$ has a unique neutral fixed end $e_{\alpha}$. Given $N_{\alpha}, N_{\beta} \in \mathscr{N}$, choose $N_{\gamma} \subset N_{\alpha} \cap N_{\beta}$; we have then

$$
\left\{e_{\alpha}\right\}=\mathscr{E}^{N_{\alpha}}=\mathscr{E}^{N_{\gamma}}=\mathscr{E}^{N_{\beta}}=\left\{e_{\beta}\right\} .
$$

Thus there is a common neutral fixed end $e$ for $\mathscr{N}$. Given $N_{\alpha} \in \mathscr{N}$, $g \in G$, choose $N_{\beta} \subset g N_{\alpha} g^{-1}$; it follows that

$$
\{e\}=\mathscr{E}^{N_{\beta}}=\mathscr{E}^{g N_{\alpha} g-1}=\{g e\}
$$

and thus $e$ is a fixed end for $G$.

Since an amalgam has elements which have no fixed points on 
the tree corresponding to the amalgamation it follows from Corollary 3 and Theorem 2 that there can be no fixed end for this action. Similarly, for an HNN extension $A_{c}^{*}(C \neq A)$ acting on its corresponding tree there can be no fixed end. To see this, we may choose without loss of generality a representative half-line for this end with initial vertex and edge having stabilizers $A$ and $C$ respectively; then for $g \in A$ it follows from Theorem 4 that $g$ is neutral on this half-line and thus $g \in C$, whence $C=A$. We shall use these remarks together with Proposition 5 to derive some important consequences for topological groups. Also, this proposition will provide useful information if the family consists of a single normal subgroup.

As a further remark on extensions of groups having property $\mathrm{FA}^{\prime}$ we have the following result.

THEOREM 5. If $H$ and $K$ are subgroups of $G$ having property $\mathrm{FA}^{\prime}$ and $G=H K$ then $G$ has property $\mathrm{FA}^{\prime}$.

Proof. Let $g \in G$ be written as $g=h k, h \in H, k \in K$; express now $k h=h^{\prime} k^{\prime}, h^{\prime} \in H, k^{\prime} \in K$. Thus we have

$$
\left(h^{-1} h^{\prime}\right) k^{\prime}\left(h^{-1} k^{-1} h\right)=1 .
$$

By results of Serre [6, Corollary 1 to Proposition 26] we can find a common fixed point $P \in X$ of the automorphisms $h^{-1} h^{\prime}, k^{\prime}, h^{-1} k^{-1} h$ for an action of $G$ on the tree $X$ if each has a fixed point; this is so from the $\mathrm{FA}^{\prime}$ hypothesis for $H$ and $K$. Consequently, we have the properties:

$$
h P=h^{\prime} P, k^{\prime} P=P, k(h P)=h P .
$$

Let $X^{k}, X^{k^{\prime}}$ be the trees of fixed points of $k$ and $k^{\prime} ; X^{k} \cap X^{k^{\prime}} \neq \varnothing$ since $K$ has property $\mathrm{FA}^{\prime}$ (condition (ii)). Since $P \in X^{k^{\prime}}, h P \in X^{k}$, it follows that the midpoint $Q$ of the geodesic from $P$ to $h P$ is fixed by $h$ [6, Corollary 2 to Proposition 23] and also by $h^{\prime}$ since $h P=h^{\prime} P$; thus $Q \in X^{k}$ or $Q \in X^{k^{\prime}}$. If $Q \in X^{k}$ then $h k Q=Q$. If $Q \in X^{k^{\prime}}$ then $h^{\prime} k^{\prime} Q=$ $Q$; but $h k=h(k h) h^{-1}=h\left(h^{\prime} k^{\prime}\right) h^{-1}$ so $h k(Q)=h k(h Q)=h(k h) h^{-1}(h Q)=$ $h h^{\prime} k^{\prime} Q=h Q=Q$. Hence $g$ has a fixed point for its action on $X$.

3. We now derive consequences for topological groups from the results of the previous sections.

THEOREM 6. If $G$ is a connected locally compact topological group then $G$ is of type $\mathrm{FA}^{\prime}$.

Proof. As a first step we decompose $G$ as 


$$
G=L C R
$$

where $L$ is a semisimple (connected) Lie subgroup, $C$ is a compact connected semisimple subgroup and $R$ is the radical of $G$ (maximal solvable connected closed normal subgroup) and $C R$ is a closed normal subgroup [5, Theorem 1]. [One uses the solution of Hilbert's fifth problem to see the equivalence of connected locally compact and Iwasawa's notion of $(L)$ group [3].] Now using [4, Lemma 3.12] we decompose the group $L$ as $L=H M$ where $H$ is a connected solvable Lie group and $M$ is either the maximal compact subgroup $K$ of $L$ or $M=K \times V$ where $V$ is a vector group. It suffices then using Theorem 5 to verify that $H, M, C, R$ are of type $\mathrm{FA}^{\prime}$. Compact groups are of type $\mathrm{FA}^{\prime}$ [1]; also any vector group being divisible and abelian is $\mathrm{FA}^{\prime}$. It remains to show that a connected solvable group $S$ is of type $\mathrm{FA}^{\prime}$. Using Iwasawa's decomposition of a locally compact connected group as

$$
G=H_{1} H_{2} \cdots H_{r} K
$$

where $K$ is maximal compact and $H_{i} \cong \boldsymbol{R} 1 \leqq i \leqq v$ [4; Theorem 13], we see that $G$ has no nontrivial homomorphisms to $Z$. Now if $S$ is an amalgam then using Bass' result for solvable groups [2, Theorem 6.1] we obtain a surjective homomorphism $S \stackrel{\varphi}{\rightarrow} \boldsymbol{Z}_{2}^{*} \boldsymbol{Z}_{2}$. However using the Iwasawa decomposition above for $S(=G)$ we obtain $\left.\varphi\right|_{H_{i}},\left.\varphi\right|_{K}$ are trivial homomorphisms. To see this notice that $H_{i}, K$ are of type FA $^{\prime}$ and hence by Proposition 2 each of the restrictions has image in a conjugate of one of the $Z_{2}$ factors; the divisibility of $H_{i} 1 \leqq i \leqq r, K$ then forces each image to be trivial and thus also $\varphi$.

COROLlaRY 1. If $G$ is a locally compact topological group with $G / G_{0}$ compact then $G$ is of type $\mathrm{FA}^{\prime}$.

Proof. This follows immediately from the theorem above, Corollary 2 to Theorem 4 and main result of [1].

Corollary 2. Suppose $G$ is a locally compact topological group. If $G$ is an amalgam, $G=A_{C}^{*} B$, or an $\mathrm{HNN}$ extension, $G=A_{C}^{*}$, then $G_{0} \subset C$.

Proof. The connected component of the identity $G_{0}$ is of type $F^{\prime}$. Using Proposition 5 and the remarks following it we see that $G_{0}$ has a fixed point for its action on the tree corresponding to the amalgam or the HNN extension if $C \neq A$. Since $G_{0}$ is normal we see immediately that $G_{0} \subset C$. In case the HNN extension has $C=A$ the corresponding tree has a fixed end, say $e$; using Theorem 4 now, 
each element of $G_{0}$ has a fixed point so $G_{0} \subset \operatorname{ker} v_{e}=A$.

CoRollary 3. Suppose $G$ is a locally compact topological group. If $G$ is an amalgam, $G=A_{C}^{*} B$ or an $\mathrm{HNN}$ extension, $G=A_{C}^{*}$, then $C$ is open in $G$.

Proof. Without loss of generality we may replace $G$ by $G / G_{0}$ using Corollary 2 above and assume then that $G$ is a locally compact totally disconnected topological group. It is well known that $G$ has a neighborhood basis of the identity given by compact open subgroups [Hewitt and Ross, Abstract Harmonic Analysis, p. 62]. Since compact groups are of type $\mathrm{FA}^{\prime}$ this is a normal filtering family of type $\mathrm{FA}^{\prime}$; by dint of Proposition 5 then some compact open subgroup $U$ has a fixed point. Without loss of generality we may assume $U \subset A$. In case $G$ is an amalgam choose $g \in B-C$ so that $U \cap g U g^{-1} \subset$ $A \cap g A g^{-1} \subset C$; hence $C$ is open. If $G$ is an $\mathrm{HNN}$ extension it is generated by $A$ together with an element $t$ (which generates the fundamental group of $X / G[6, \mathrm{p} .62])$; thus

$$
U \cap t U t^{-1} \subset A \cap t A t^{-1} \subset C \text {. }
$$

For the HNN extension to which Proposition 5 doesn't apply, viz. $G=A_{A}^{*}$ we notice as in the proof of Corollary 2 that there is a fixed end $e$ and hence for any compact open subgroup $U$,

$$
U \subset \operatorname{ker} v_{e} \subset A
$$

since $U$ is of type $\mathrm{FA}^{\prime}$.

ACKNOWLEDgments. The author wishes to thank H. Bass and K. Moss for fruitful discussions during the preparation of this paper.

\section{REFERENCES}

1. R. Alperin, Compact groups acting on trees, to appear in Houston J. Math.

2. H. Bass, Some remarks on group actions on trees, Communications in Algebra, 4 (12), (1976), 1091-1126.

3. V. M. Gluskov, Locally compact groups and Hilbert's fifth problem, Amer. Math. Soc. Trans. Series 2, 15 (1960), 55-94.

4. K. Iwasawa, Some types of topological groups, Annals of Math., 50 (1949), 507-588.

5. Y. Matsushima, On the decomposition of an (L)-group, Math. Soc. of Japan, 1 (1950), 264-274.

6. J.-P. Serre, Arbres, Amalgames, $S L_{2}$, Asterisque 46, Societé Mathematique de France, (1977).

7. J. Tits, A theorem of Lie-Kolchin for trees, in Contributions to Algebra: A Collection of Papers Dedicated to Ellis Kolchin, pp. 377-388, Academic Press, (1977).

8. - Sur le group des automorphismes d'un arbre, Essays on Topology and related topics, Mémoires dédiés à Georges de Rham, Springer-Verlag, pp. 188-211, (1970). 
Received May 14, 1980 and in revised form September 30, 1980. Partially supported by NSF Grant 79-03053.

The University of OKLahoma

NoRMAN, OK 73019 


\section{PACIFIC JOURNAL OF MATHEMATICS}

\section{EDITORS}

DONALD BABBITT (Managing Editor)

University of California

Los Angeles, California 90024

Hugo RossI

University of Utah

Salt Lake City, UT 84112

C. C. MOORE and ARTHUR Agus

University of California

Berkeley, CA 94720

\section{J. DUGUNDJI}

Department of Mathematics University of Southern California Los Angeles, California 90007

R. FinN and J. MILGRAM Stanford University Stanford, California 94305

\section{ASSOCIATE EDITORS}
R. ARNES
E. F. BeCKENBACH
B. H. Neumann
F. WOLF
K. YosHIDA

\section{SUPPORTING INSTITUTIONS}

UNIVERSITY OF ARIZONA

UNIVERSITY OF BRITISH COLUMBIA

CALIFORNIA INSTITUTE OF TECHNOLOGY

UNIVERSITY OF CALIFORNIA

MONTANA STATE UNIVERSITY

UNIVERSITY OF NEVADA, RENO

NEW MEXICO STATE UNIVERSITY

OREGON STATE UNIVERSITY
UNIVERSITY OF OREGON UNIVERSITY OF SOUTHERN CALIFORNIA STANFORD UNIVERSITY UNIVERSITY OF HAWAII UNIVERSITY OF TOKYO UNIVERSITY OF UTAH WASHINGTON STATE UNIVERSITY UNIVERSITY OF WASHINGTON 


\section{Pacific Journal of Mathematics}

Vol. 100, No. $1 \quad$ September, 1982

Charalambos D. Aliprantis, Owen Sidney Burkinshaw and M. Duhoux,

Compactness properties of abstract kernel operators $\ldots \ldots \ldots \ldots \ldots \ldots 1$

Roger C. Alperin, Locally compact groups acting on trees .............23

Robert F. Brown, Real homology of Lie group homomorphisms ......... 33

Karen Chase, Maximal groups in sandwich semigroups of binary relations . . 43

W. Wistar (William) Comfort and T. Soundararajan, Pseudocompact

group topologies and totally dense subgroups $\ldots \ldots \ldots \ldots \ldots \ldots \ldots 61$

M. Ferri and C. Gagliardi, Crystallisation moves $\ldots \ldots \ldots \ldots \ldots \ldots \ldots$

Kenneth R. Goodearl, Directly finite aleph-nought-continuous regular

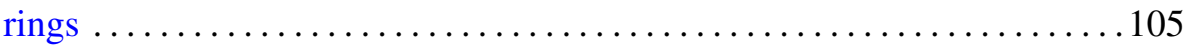

Edward Lewis Green, On the representation theory of rings in matrix

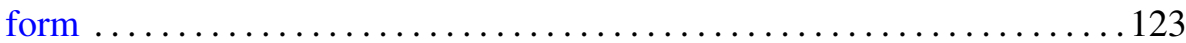

Walter Hengartner and Glenn E. Schober, Interpolation, continuation, and

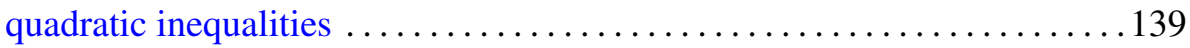

Kenneth Kunen and Haskell Paul Rosenthal, Martingale proofs of some geometrical results in Banach space theory $\ldots \ldots \ldots \ldots \ldots \ldots \ldots \ldots \ldots \ldots$

Brian William McEnnis, Shifts on indefinite inner product spaces. II . . . . . 177

Roman Pol, Note on the spaces $P(S)$ of regular probability measures whose topology is determined by countable subsets $\ldots \ldots \ldots \ldots \ldots \ldots \ldots \ldots 185$

Joan Manuel Verdera Melenchón, Finitely generated projective extensions

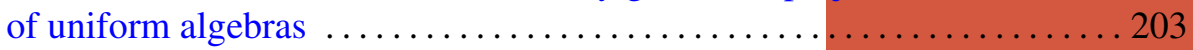

Cheng Ye You, Fixed point classes of a fiber map .................. 217 